\title{
DAVIS-TYPE THEOREMS FOR MARTINGALE DIFFERENCE SEQUENCES
}

\author{
GEORGE STOICA
}

Received 25 February 2004 and in revised form 10 August 2004

We study Davis-type theorems on the optimal rate of convergence of moderate deviation probabilities. In the case of martingale difference sequences, under the finite $p$ th moments hypothesis $(1 \leq p<\infty)$, and depending on the normalization factor, our results show that Davis' theorems either hold if and only if $p>2$ or fail for all $p \geq 1$. This is in sharp contrast with the classical case of i.i.d. centered sequences, where both Davis' theorems hold under the finite second moment hypothesis (or less).

\section{Introduction}

Let $\left(X_{n}\right)_{n \geq 1}$ be a sequence of random variables on a probability space $(\Omega, \mathscr{F}, P)$ and, for each $n \geq 1$, denote by $\mathscr{F}_{n}$ the $\sigma$-algebra generated by $X_{1}, X_{2}, \ldots, X_{n}$. We say that $\left(X_{n}\right)_{n \geq 1}$ is martingale difference sequence if $S_{n}:=X_{1}+\cdots+X_{n}$ is a martingale with respect to the filtration $\left(\mathscr{F}_{n}\right)_{n \geq 1}$, that is, $E\left[S_{n} \mid \mathscr{F}_{n-1}\right]=S_{n-1}$ a.s. for $n \geq 1$ (here $S_{0}=0$ and $\mathscr{F}_{0}$ is the trivial $\sigma$-algebra). Obviously, any i.i.d. centered sequence $\left(X_{n}\right)_{n \geq 1}$ from $L^{1}$ is a martingale difference sequence, but the converse is false (consider, e.g., ergodic dynamical systems of positive entropy). Let $p \geq 1$; we say that the martingale difference sequence $\left(X_{n}\right)_{n \geq 1}$ is $L^{p}$-bounded if it has finite $p$ th moments, that is, $\left\|X_{n}\right\|_{p} \leq C$ for some constant $C>0$ and any $n \geq 1$.

Davis' first and second theorems refer to the rate of convergence of the moderate deviations probabilities $P\left[\left|S_{n}\right|>\varepsilon(n \log n)^{1 / 2}\right]$ or $P\left[\left|S_{n}\right|>\varepsilon(n \log \log n)^{1 / 2}\right]$, for $\varepsilon>0$; they were studied for $L^{2}$-bounded i.i.d. sequences in Davis [1, 2] (see also $[3,4,5]$ ). In contrast with the i.i.d. case, in this paper, we prove that Davis' first theorem for $L^{p}$-bounded martingale difference sequences holds if and only if $p>2$. Depending on the normalization factor therein, we prove that Davis' second theorem for $L^{p}$-bounded martingale difference sequences holds if and only if $p>2$, or fails for any $p \geq 1$. Our results complement the optimal rates of convergence in the Baum-Katz-type theorems for martingale difference sequences obtained in $[6,7]$, that is, the rate of convergence of the large deviations probabilities $P\left[\left|S_{n}\right|>\varepsilon n^{\alpha}\right]$, with $1 / 2<\alpha \leq 1$. Moreover, sharp precise asymptotics (as $\varepsilon \backslash 0$ ) are known for i.i.d. centered sequences in both Baum-Katz and Davis' theorems (see $[4,5])$. 
In the martingale difference case, our proofs of Davis' theorems will also provide, as consequence, such asymptotics.

\section{Davis' first theorem}

Let $\varepsilon>0$ and let $\delta=\delta(p)$ be a function of $p \geq 1$. Consider the series

$$
\sum_{n=2}^{\infty} \frac{(\log n)^{\delta}}{n} P\left[\left|S_{n}\right|>\varepsilon(n \log n)^{1 / 2}\right] .
$$

Assume $\left(X_{n}\right)_{n \geq 1}$ is an i.i.d. sequence and $\delta=1$. Davis' first theorem says that series (2.1) is convergent for any $\varepsilon>0$ if and only if $\left(X_{n}\right)_{n \geq 1}$ is an $L^{2}$-bounded centered sequence (see $[1,3,4])$.

In the martingale difference sequences case, we have the following result.

Theorem 2.1. (i) For any $p>2$ and $L^{p}$-bounded martingale difference sequence $\left(X_{n}\right)_{n \geq 1}$, series (2.1) is convergent for any $0 \leq \delta<p / 2-1$ and any $\varepsilon>0$.

(ii) For any $p \geq 1$ there exists an $L^{p}$-bounded martingale difference sequence $\left(X_{n}\right)_{n \geq 1}$ such that series (2.1) diverges for any $\delta>p / 2-1$ and any $\varepsilon>0$.

(iii) There exist a probability space $(\Omega, \mathscr{F}, P)$ and an $L^{p-\lambda}$-bounded martingale difference sequence $\left(X_{n}\right)_{n \geq 1}$ (for all $0<\lambda \leq p-1$ ) such that series (2.1) diverges for $\delta \geq p / 2-1$ and any $\varepsilon>0$.

Proof. Throughout the paper, $C>0$ denotes a generic numerical constant. The sign between two series means that they are either both convergent or both divergent. In the sequel we use that each of the series

$$
\sum_{n=2}^{\infty} \frac{(\log n)^{\alpha}}{n^{\beta}}, \quad \sum_{n=3}^{\infty} \frac{(\log \log n)^{\alpha}}{n(\log n)^{\beta}}
$$

is convergent if and only if $\beta>1$ or $\beta=1$ and $\alpha<-1$ (by the integral test).

The idea in proving Theorem 2.1(i) is to obtain direct sharp estimates for the twosided deviation probabilities $P\left[\left|X_{n}\right|-(\log n)^{a}\right]$ for some $a>0$ to be specified later, instead of using standard remainder term estimates for the central limit theorem. To this aim, we are going to use the following truncated processes from [6, Theorem 3.2] adapted to our problem:

$$
\begin{aligned}
& X_{n}^{1}:=X_{n} \mathbf{1}_{\left\{\left|X_{n}\right| \leq(\log n)^{a}\right\}}-E\left[X_{n} \mathbf{1}_{\left\{\left|X_{n}\right| \leq(\log n)^{a}\right\}} \mid \mathscr{F}_{n-1}\right], \\
& X_{n}^{2}:=X_{n} \mathbf{1}_{\left\{\left|X_{n}\right|>(\log n)^{a}\right\}}-E\left[X_{n} \mathbf{1}_{\left\{\left|X_{n}\right|>(\log n)^{a}\right\}} \mid \mathscr{F}_{n-1}\right],
\end{aligned}
$$

where $\left(\mathscr{F}_{n}\right)_{n \geq 1}$ is the filtration generated by $\left(X_{n}\right)_{n \geq 1}$, and $\mathscr{F}_{0}$ is the trivial $\sigma$-algebra. It is immediate that $\left(X_{n}^{1}\right)_{n \geq 1}$ and $\left(X_{n}^{2}\right)_{n \geq 1}$ are martingale difference sequences with respect to $\left(\mathscr{F}_{n}\right)_{n \geq 1}$ and $X_{n}=X_{n}^{1}+X_{n}^{2}$. Put $S_{n}^{i}=X_{1}^{i}+\cdots+X_{n}^{i} ; i=1,2$.

Let $p>2$; as $\left\|X_{k}\right\|_{p} \leq C$, we have, for any $k \geq 1$ and $x>0$,

$$
F_{k}(x):=P\left[\left|X_{k}\right|>x\right] \leq C x^{-P} .
$$


We obtain

$$
\begin{aligned}
E\left|X_{k}^{2}\right|^{2} & \leq-\int_{(\log n)^{a}}^{+\infty} x^{2} d F_{k}(x) \\
& =-\lim _{N \rightarrow+\infty}\left[N^{2} F_{k}(N)-(\log n)^{2 a} F_{k}\left((\log n)^{a}\right)+2 \int_{(\log n)^{a}}^{N} x F_{k}(x) d x\right] \\
& \leq C(\log n)^{(2-p) a} .
\end{aligned}
$$

The second line is obtained by integration by parts and by using estimation (2.4) in the proper integral in (2.5).

By a general property for the martingale difference sequences, we have

$$
E\left|S_{n}^{2}\right|^{2}=\sum_{k=1}^{n} E\left|X_{k}^{2}\right|^{2}
$$

Combining (2.5) and (2.6) gives

$$
\begin{aligned}
P\left[\left|S_{n}^{2}\right|>\frac{\varepsilon}{2}(n \log n)^{1 / 2}\right] & =P\left[\left|S_{n}^{2}\right|^{2}>\frac{\varepsilon^{2}}{4} n \log n\right] \\
& \leq C \varepsilon^{-2}(n \log n)^{-1} E\left|S_{n}^{2}\right|^{2} \\
& \leq C \varepsilon^{-2}(\log n)^{(2-p) a-1} .
\end{aligned}
$$

Let $\alpha>2$; by [6, Theorem 3.6], and taking into account that $\left|X_{n}^{1}\right| \leq 2(\log n)^{a}$, we obtain

$$
E\left|S_{n}^{1}\right|^{\alpha} \leq C n^{\alpha / 2-1} \sum_{k=1}^{n} E\left|X_{k}^{1}\right|^{\alpha} \leq C n^{\alpha / 2}(\log n)^{a \alpha} .
$$

Hence

$$
P\left[\left|S_{n}^{1}\right|^{\alpha}>\left(\frac{\varepsilon}{2}\right)^{\alpha}(n \log n)^{\alpha / 2}\right] \leq C \varepsilon^{-\alpha}(n \log n)^{-\alpha / 2} E\left|S_{n}^{1}\right|^{\alpha} \leq C \varepsilon^{-\alpha}(\log n)^{a \alpha-\alpha / 2} .
$$

Let $0 \leq \delta<p / 2-1$. In the sequel take $\alpha>\alpha_{0}:=2(p-2)(1+\delta) /(p-2-2 \delta)$, and note that $\alpha_{0} \geq 2$. By (2.7) and (2.9) we obtain that series (2.1) is dominated by

$$
\begin{gathered}
\sum_{n=2}^{\infty} \frac{(\log n)^{\delta}}{n} P\left[\left|S_{n}^{1}\right|>\frac{\varepsilon}{2}(n \log n)^{1 / 2}\right]+\sum_{n=2}^{\infty} \frac{(\log n)^{\delta}}{n} P\left[\left|S_{n}^{2}\right|>\frac{\varepsilon}{2}(n \log n)^{1 / 2}\right] \\
\leq C \varepsilon^{-\alpha} \sum_{n=2}^{\infty} \frac{(\log n)^{a \alpha-\alpha / 2+\delta}}{n}+C \varepsilon^{-2} \sum_{n=2}^{\infty} \frac{(\log n)^{(2-p) a-1+\delta}}{n}=: A+B .
\end{gathered}
$$

Part (i) of Theorem 2.1 is proved if we choose $\delta /(p-2)<a<(\alpha-2 \delta-2) / 2 \alpha$. Indeed, the first inequality ensures the convergence of series $B$, and the second inequality ensures the convergence of series A. Such an $a$ exists, because the compatibility inequality $\delta /(\mathrm{p}-2)<(\alpha-2 \delta-2) / 2 \alpha$ is equivalent to $\alpha>\alpha_{0}$. 
For part (ii) of Theorem 2.1, let $p \geq 1$ and $\delta>p / 2-1$. We need to construct a martingale difference sequence $\left(X_{n}\right)_{n \geq 1}$ with $\left\|X_{n}\right\|_{p} \leq C$ for any $n \geq 1$, and such that series (2.1) diverges. To this aim, consider $X_{n}=Z \cdot Y_{n}$, where $\left(Y_{n}\right)_{n \geq 1}$ is an i.i.d. bounded centered sequence, and $Z$ is independent of $\left(Y_{n}\right)_{n \geq 1}$ with

$$
P[|Z|>n]=C n^{-c}
$$

for $n \geq 1$ (where $C$ is a normalization factor), for some $c>0$ to be specified later. If $c>p$, then $\left(X_{n}\right)_{n \geq 1}$ has finite $p$ th moments, as

$$
E\left|X_{n}\right|^{p} \leq C E|Z|^{p} \leq C \sum_{n=1}^{\infty} n^{p}(P[|Z|>n]-P[|Z|>n+1]) \sim \sum_{n=1}^{\infty} n^{p-c-1} .
$$

By independence and the central limit theorem, we have

$$
\begin{aligned}
P\left[\left|S_{n}\right|>\varepsilon(n \log n)^{1 / 2}\right] & \geq P\left[\left|Y_{1}+\cdots+Y_{n}\right|>n^{1 / 2}\right] \cdot P\left[|Z|>\varepsilon(\log n)^{1 / 2}\right] \\
& \geq C P\left[|Z|>\varepsilon(\log n)^{1 / 2}\right]
\end{aligned}
$$

hence

$$
\sum_{n=2}^{\infty} \frac{(\log n)^{\delta}}{n} P\left[\left|S_{n}\right|>\mathcal{E}(n \log n)^{1 / 2}\right] \geq C \sum_{n=2}^{\infty} n^{-1}(\log n)^{\delta-c / 2}
$$

The latter series in (2.14) diverges if $\delta \geq c / 2-1$. Hence, for series (2.1) to diverge, it suffices to choose $p<c \leq 2 \delta+2$. Such $c$ exists because the compatibility inequality $p<$ $2 \delta+2$ is equivalent to $\delta>p / 2-1$.

Let $\delta \geq p / 2-1$; to prove Theorem 2.1(iii) consider $Z$ with finite second moment and such that

$$
P[|Z|>n] \geq \frac{C}{n^{p}}
$$

for $n \geq 1$. Define as in the proof of part (ii) $X_{n}=Z \cdot Y_{n}$ and note that $\left(X_{n}\right)_{n \geq 1}$ has finite moments of order $p-\lambda$, for all $0<\lambda \leq p-1$. As such,

$$
\begin{aligned}
& \sum_{n=2}^{\infty} \frac{(\log n)^{\delta}}{n} P\left[\left|S_{n}\right|>\varepsilon(n \log n)^{1 / 2}\right] \\
& \quad \geq C \sum_{n=2}^{\infty} \frac{(\log n)^{\delta}}{n} P\left[|Z|>\varepsilon(\log n)^{1 / 2}\right] \geq C \sum_{n=2}^{\infty} \frac{(\log n)^{\delta-p / 2}}{n}=+\infty
\end{aligned}
$$


Remark 2.2. In particular, from Theorem 2.1, we deduce the following. If $1 \leq p \leq 2$, then series (2.1) diverges for any $\delta \geq 0$ and some $L^{p}$-bounded martingale difference sequence $\left(X_{n}\right)_{n \geq 1}$. For any $p>2$ and $L^{p}$-bounded martingale difference sequence $\left(X_{n}\right)_{n \geq 1}$, series (2.1) converges for some $\delta>0$ : if $p>3$ one can take $\delta=1 / 2$, and if $p>4$ one can take $\delta=1$, and so forth.

The asymptotics, as $\varepsilon \succ 0$, in Theorem 2.1(i) are given below.

Corollary 2.3. For any $p>2$ and $L^{p}$-bounded martingale difference sequence $\left(X_{n}\right)_{n \geq 1}$, it holds that

$$
\lim _{\varepsilon \backslash 0} \varepsilon^{\alpha_{0}} \sum_{n=2}^{\infty} \frac{(\log n)^{\delta}}{n} P\left[\left|S_{n}\right|>\varepsilon(n \log n)^{1 / 2}\right]<+\infty
$$

for any $0 \leq \delta<p / 2-1$, and where $\alpha_{0}:=2(p-2)(1+\delta) /(p-2-2 \delta)$.

Proof. In formula (2.10), as $\varepsilon \succ 0$, series B behaves like $\varepsilon^{-2}$ and series A behaves like $\varepsilon^{\alpha}$, for $\alpha>\alpha_{0}$. Notice that $\alpha_{0} \geq 2 \delta+2 \geq 2$, hence the normalization factor $\varepsilon^{\alpha_{0}}$ makes the limit in formula (2.17) finite.

Remark 2.4. For $L^{2}$-bounded centered i.i.d. sequences, the optimal normalization factor in Corollary 3.2 is $\varepsilon^{2 \delta+2}$ (see [4, Theorem 3]), in which case the limit in (2.17) is strictly positive. Moreover, note that $\alpha_{0} \geq 2 \delta+2 \geq 2$ and $\lim \alpha_{0}=2 \delta+2$ as $p \rightarrow \infty$, hence the latter result can be viewed as a limiting case of our general result (2.17).

\section{Davis' second theorem}

Let $\varepsilon>0$ and let $\delta=\delta(p)$ be a function of $p \geq 1$. Consider the series

$$
\sum_{n=3}^{\infty} \frac{1}{n(\log n)^{\delta}} P\left[\left|S_{n}\right|>\varepsilon(n \log \log n)^{1 / 2}\right] .
$$

Assume $\left(X_{n}\right)_{n \geq 1}$ is an i.i.d. sequence. If $\delta=0$, Davis' second theorem says that series (3.1) converges when $\varepsilon>\sigma \sqrt{2}$ if and only if $\left(X_{n}\right)_{n \geq 1}$ is an $L^{2}$-bounded centered sequence, and where $\sigma^{2}:=E\left[X_{1}^{2}\right]$. If $\delta=1$, series (3.1) is convergent for any $\varepsilon>0$ if $\left(X_{n}\right)_{n \geq 1}$ is centered and satisfies slightly less than a second moment, but the necessary and sufficient moment condition is not known (see $[2,3,4,5]$ ).

In the martingale difference sequences case, we have the following result.

Theorem 3.1. (i) For any $p>2$ and $L^{p}$-bounded martingale difference sequence $\left(X_{n}\right)_{n \geq 1}$, series (3.1) is convergent for $\delta \geq 1$ and any $\varepsilon>0$.

(ii) For any $1 \leq p<2$ there exists an $L^{p}$-bounded martingale difference sequence $\left(X_{n}\right)_{n \geq 1}$ such that series (3.1) diverges for $\delta=1$ and any $\varepsilon>0$.

(iii) For any $p \geq 1$ there exists an $L^{p}$-bounded martingale difference sequence $\left(X_{n}\right)_{n \geq 1}$ such that series (3.1) diverges for any $0 \leq \delta<1$ and $\varepsilon>0$.

(iv) There exist a probability space $(\Omega, \mathscr{F}, P)$ and an $L^{2-\lambda}$-bounded martingale difference sequence $\left(X_{n}\right)_{n \geq 1}$ (for all $0<\lambda \leq 1$ ) such that series (3.1) diverges for $0 \leq \delta \leq 1$ and any $\varepsilon>0$. 
Proof. To prove (i), first remark that formulas (2.7) and (2.9) can be proved the same way as we did in Theorem 2.1(i) when replacing $(\log n)^{a}$ by $(\log \log n)^{a}$. As such, with the same notations therein, we obtain

$$
\begin{aligned}
& \sum_{n=3}^{\infty} \frac{1}{n(\log n)^{\delta}} P\left[\left|S_{n}\right|>\frac{\varepsilon}{2}(n \log \log n)^{1 / 2}\right] \\
& \quad \leq C \varepsilon^{-\alpha} \sum_{n=3}^{\infty} \frac{(\log \log n)^{\alpha \alpha-\alpha / 2}}{n(\log n)^{\delta}}+C \varepsilon^{-2} \sum_{n=3}^{\infty} \frac{(\log \log n)^{(2-p) a-1}}{n(\log n)^{\delta}} .
\end{aligned}
$$

From (3.2) we deduce that series (3.1) with $\delta>1$ is convergent regardless of the values of $a$ and $\alpha$; also, series (3.1) with $\delta=1$ is convergent if $0<a<(\alpha-2) / 2 \alpha$. Indeed, in the latter case, the second series in the second line of (3.2) is convergent if $a>0$ and the first series in the second line of (3.2) is convergent if $a<(\alpha-2) / 2 \alpha$. Such $a$ exists because $\alpha>2$.

For parts (ii) and (iii) of Theorem 3.1, within the same construction of the counterexample of martingale difference sequence in Theorem 2.1(ii), take $Z$ with $P[|Z|>n]=$ $C n^{-c}$ for some $c>p$. Note that $\left(X_{n}\right)_{n \geq 1}$ have finite $p$ th-order moments. As

$$
\begin{aligned}
P\left[\left|S_{n}\right|>\varepsilon(n \log \log n)^{1 / 2}\right] & \geq P\left[\left|Y_{1}+\cdots+Y_{n}\right|>n^{1 / 2}\right] \cdot P\left[|Z|>\varepsilon(\log \log n)^{1 / 2}\right] \\
& \geq C \cdot P\left[|Z|>\varepsilon(\log \log n)^{1 / 2}\right],
\end{aligned}
$$

series (3.1) dominates the following analog of (2.14):

$$
C \sum_{n=3}^{\infty} \frac{1}{n(\log n)^{\delta}} P\left[|Z|>\varepsilon(\log \log n)^{1 / 2}\right] \geq C \sum_{n=3}^{\infty} \frac{(\log \log n)^{-c / 2}}{n(\log n)^{\delta}} .
$$

The latter series in (3.4) diverges for any $0 \leq \delta<1$ and $c>0$, and this proves (iii); it also diverges for $\delta=1$ and $c<2$, and this proves (ii) for $1 \leq p<2$.

To prove (iv) consider $Z$ with finite second moment and such that $P[|Z|>n] \geq C / n^{2}$ for $n \geq 1$. Define as above $X_{n}=Z \cdot Y_{n}$ and note that $\left(X_{n}\right)_{n \geq 1}$ has finite moments of order $2-\lambda$, for all $0<\lambda \leq 1$. As such,

$$
\begin{aligned}
\sum_{n=3}^{\infty} \frac{1}{n(\log n)^{\delta}} P\left[\left|S_{n}\right|>\varepsilon(n \log \log n)^{1 / 2}\right] & \geq C \sum_{n=3}^{\infty} \frac{1}{n(\log n)^{\delta}} P\left[|Z|>\varepsilon(\log \log n)^{1 / 2}\right] \\
& \geq C \sum_{n=3}^{\infty} \frac{1}{n(\log n)^{\delta}(\log \log n)}=+\infty,
\end{aligned}
$$

as $0 \leq \delta \leq 1$.

The asymptotics, as $\varepsilon \searrow 0$, in Theorem 3.1(i) are given below.

Corollary 3.2. For any $p>2, \delta \geq 1$ and $L^{p}$-bounded martingale difference sequence $\left(X_{n}\right)_{n \geq 1}$, it holds that

$$
\lim _{\mathcal{E} \backslash 0} \varepsilon^{2} \sum_{n=3}^{\infty} \frac{1}{n(\log n)^{\delta}} P\left[\left|S_{n}\right|>\varepsilon(n \log n \log n)^{1 / 2}\right]<+\infty .
$$


Proof. In formula (3.2), as $\varepsilon \succ 0$, the second series behaves like $\varepsilon^{-2}$ and the first series behaves like $\varepsilon^{\alpha}$, for all $\alpha>2$. Hence the normalization factor $\varepsilon^{2}$ makes the limit in formula (3.6) finite.

Remark 3.3. For $L^{2}$-bounded centered i.i.d. sequences, the optimal normalization factor in Corollary 3.2 is precisely $\varepsilon^{2}$ (see [5]), that is, the limit in (3.6) is strictly positive. In other words, our Corollary 3.2 gives sharp rates of convergence for series (3.1).

\section{References}

[1] J. A. Davis, Convergence rates for probabilities of moderate deviations, Ann. Math. Stat. 39 (1968), 2016-2028.

[2] Convergence rates for the law of the iterated logarithm, Ann. Math. Stat. 39 (1968), $1479-1485$.

[3] A. Gut, Convergence rates for probabilities of moderate deviations for sums of random variables with multidimensional indices, Ann. Probab. 8 (1980), no. 2, 298-313.

[4] A. Gut and A. Spătaru, Precise asymptotics in the Baum-Katz and Davis laws of large numbers, J. Math. Anal. Appl. 248 (2000), no. 1, 233-246.

[5] - Precise asymptotics in the law of the iterated logarithm, Ann. Probab. 28 (2000), no. 4, $1870-1883$.

[6] E. Lesigne and D. Volný, Large deviations for martingales, Stochastic Process. Appl. 96 (2001), no. 1, 143-159.

[7] Y. Li, A martingale inequality and large deviations, Statist. Probab. Lett. 62 (2003), no. 3, 317321.

George Stoica: Department of Mathematical Sciences, University of New Brunswick, P.O. Box 5050, Saint John, NB, Canada E2L 4L5

E-mail address: stoica@unbsj.ca 


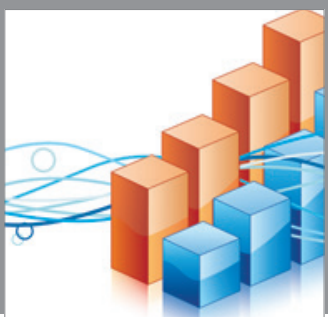

Advances in

Operations Research

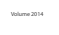

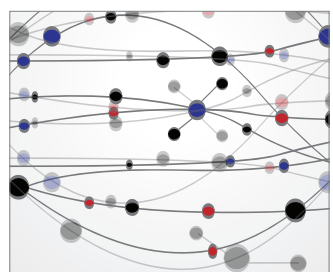

\section{The Scientific} World Journal
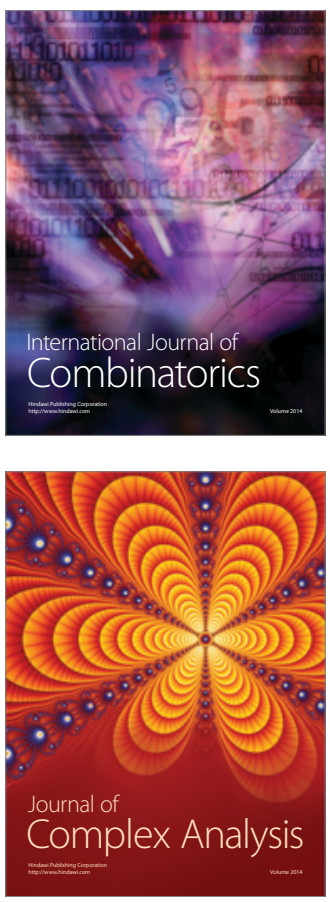

International Journal of

Mathematics and

Mathematical

Sciences
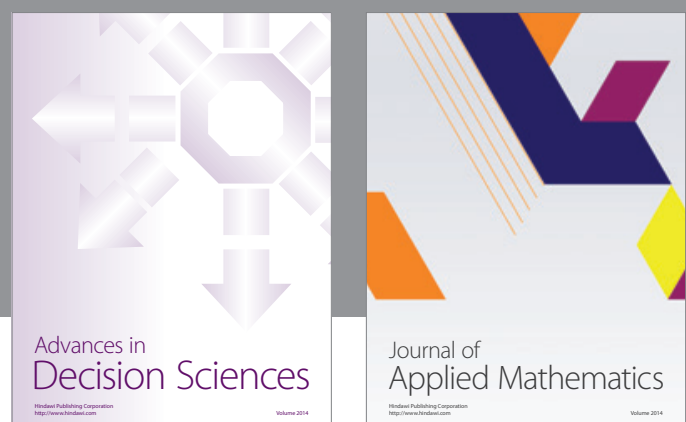

Journal of

Applied Mathematics
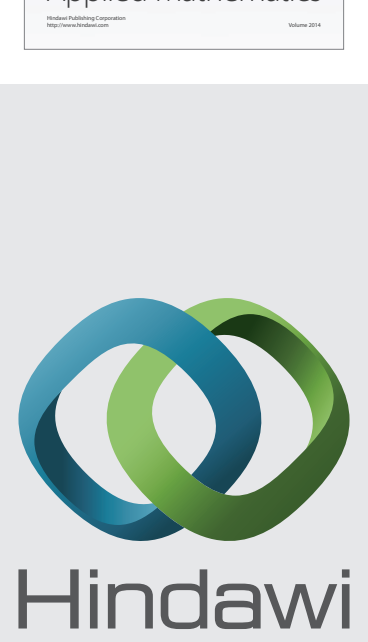

Submit your manuscripts at http://www.hindawi.com
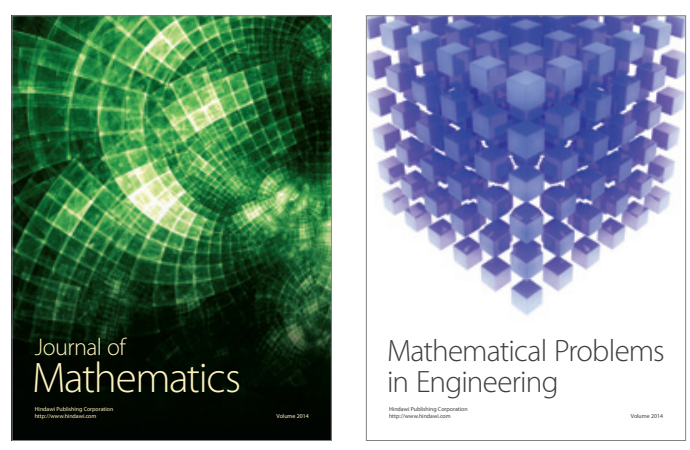

Mathematical Problems in Engineering
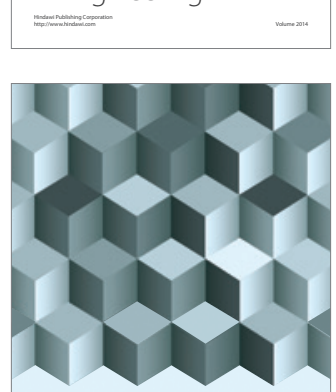

Journal of

Function Spaces
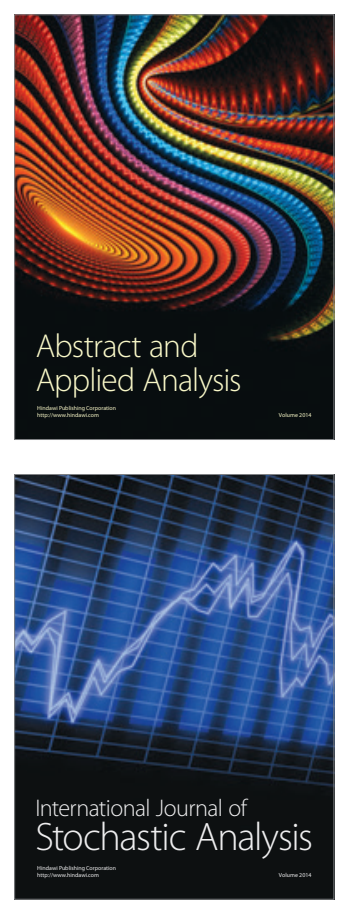

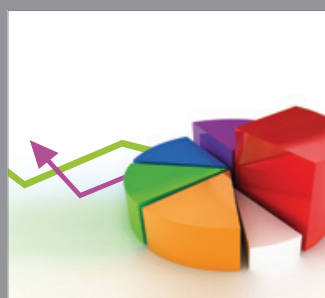

ournal of

Probability and Statistics

Promensencen
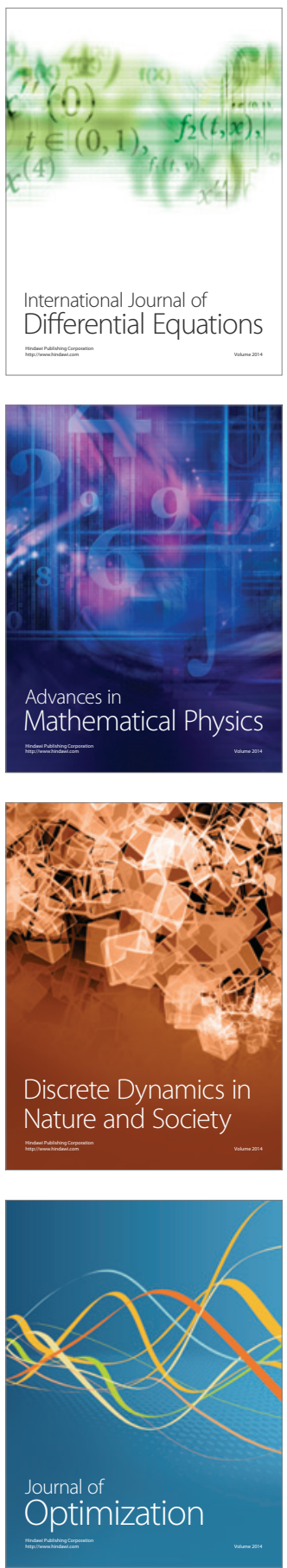